\title{
Preterm Neonatal Stage
}

National Cancer Institute

\section{Source}

National Cancer Institute. Preterm Neonatal Stage. NCI Thesaurus. Code C89888.

A human life stage that begins at birth prior to thirty-seven weeks of full gestation (full term) and continues until twenty-seven complete days after birth. 